Itinéraires Itinéraires

Littérature, textes, cultures

2011-1| 2011

Les Mémoires, une question de genre?

\title{
L'actrice, du cinéma aux arts plastiques et au roman : genre et représentation
}

\section{Thibaut Casagrande}

\section{(2) OpenEdition}

1 Journals

Édition électronique

URL : http://journals.openedition.org/itineraires/1676

DOI : $10.4000 /$ itineraires. 1676

ISSN : 2427-920X

Éditeur

Pléiade

\section{Édition imprimée}

Date de publication : 1 avril 2011

Pagination : 155-170

ISBN : 978-2-296-13692-2

ISSN : $2100-1340$

\section{Référence électronique}

Thibaut Casagrande, "L'actrice, du cinéma aux arts plastiques et au roman : genre et représentation », Itinéraires [En ligne], 2011-1 | 2011, mis en ligne le 01 avril 2011, consulté le 26 juin 2020. URL : http:// journals.openedition.org/itineraires/1676 ; DOI : https://doi.org/10.4000/itineraires.1676

\section{cc) $(1) \odot$}

Itinéraires est mis à disposition selon les termes de la licence Creative Commons Attribution - Pas d'Utilisation Commerciale - Pas de Modification 4.0 International. 


\title{
L'actrice, du cinéma aux arts plastiques et au roman : genre et représentation ${ }^{1}$
}

\begin{abstract}
This article analyzes representations of film actresses in novels and the visual arts. It questions the way this figure - a human being and a construction of cinema - is represented outside the world she belongs to, that is, outside cinema and popular culture. More specifically, it addresses the particular status of gender as a major stake in those works, from the genre of these narratives and images and the gender of those who create them, to the semiology of the actress herself. Do novelists and visual artists offer a different vision of the actress and of her often stereotypical femininity?
\end{abstract}

Keywords : gender, representation, femininity, film actress, semiology

Mots clés : genre, représentation, féminité, actrice, sémiologie

Phèdre, la " Scène de la Déclaration », la Berma avaient alors pour moi une sorte d'existence absolue. [...] Situées en retrait du monde de l'expérience courante, elles existaient par elles-mêmes, il me fallait aller vers elles [...]. L'insignifiance de [la vie] que je menais n'avait aucune importance, pas plus que les moments où on s'habille, où on se prépare pour sortir, puisque au-delà existait, d'une façon absolue, bonnes et difficiles à approcher, impossibles à posséder tout entières, ces réalités plus solides, Phèdre, la manière dont disait la Berma.

Marcel Proust, Le Côté de Guermantes I, À la recherche du temps perdu, III, Paris, Gallimard, coll. « Folio », 2001 [1920], p. 38.

1. J'aimerais remercier $\mathrm{M}^{\mathrm{me}}$ Anne Tomiche, qui a encadré les recherches dont est issu cet article, M. Pierre Zoberman, pour sa confiance et pour ses conseils, Fanny Levin, Romain Benini et Pauline Soulat pour leur attention. 
Dans ce passage situé au début du Côté de Guermantes, le narrateur d'À la Recherche du temps perdu évoque sa fascination pour la pièce de Racine et pour son interprète principale dans le roman, la Berma, célèbre comédienne de théâtre. Depuis plusieurs années, la critique a mis en évidence la place tenue dès la fin du XVIII ${ }^{e}$ siècle par les figures de la comédienne dans le roman et dans les arts plastiques, qui tendent à faire d'elle une figure privilégiée, emblème d'une certaine féminité. Cependant, dans la société, la comédienne est aujourd'hui éclipsée par l'actrice, dont la présence est accrue dans les médias. Certains romanciers et artistes contemporains, en France, en Angleterre et aux États-Unis, se sont ainsi emparés de cette figure hybride, être réel et construction du cinéma, pour en faire un personnage de roman ou le modèle de portraits, souvent dans un écart par rapport aux images générées par le cinéma ${ }^{2}$.

Le genre sexuel, entendu comme l'anglais gender ${ }^{3}$, joue un rôle central dans cette prise de distances. Héroïne de récits d'amour magnifiée par des images choisies, l'actrice est un modèle idéal qui obéit à d'intenses déterminations de genre et dont les apparitions touchent parfois au stéréotype. Les artistes et les romanciers, par le filtre d'une image autre ou d'une fiction, soumettent cependant cette figure à une représentation seconde souvent critique. C'est cette vision du genre incarnée par l'actrice de cinéma, vue par le roman et les arts plastiques, que nous aimerions évoquer, selon la méthodologie des comparative cultural studies. Il s'agit de comprendre dans quelle mesure le genre entre en jeu dans ces œuvres, chez celui qui dépeint comme dans l'objet figuré. Cela requiert ainsi une sémiologie comparée, afin d'analyser quelles conceptions du genre proposent ces investissements de la figure de l'actrice, entre romans et arts plastiques ${ }^{4}$.

2. Ces images sont nombreuses et comprennent toutes celles que le cinéma, art et industrie, génère : des films aux photographies de studio ou de presse, aux représentations non visuelles des interviews, et des articles biographiques ou critiques. C'est l'ensemble de ces signes qu'étudient les star studies, dans la lignée de l'essai séminal d'Edgar Morin publié en 1957, Les Stars (Paris, Seuil). Richard Dyer est l'auteur d'un ouvrage intitulé Stars qui a également fait date dans ce champ d'étude. On peut également citer les travaux des films studies, particulièrement marqués par l'essai de Laura Mulvey publié en 1975, « Visual Pleasure and Narrative Cinema », développement d'une analyse féministe et lacanienne de la fétichisation de l'actrice dans le cinéma hollywoodien par le «male gaze », le regard masculin.

3. On oppose traditionnellement sexe, donné naturel, et genre, construction sociale.

4. Bien que le cinéma lui-même se soit penché sur la figure de l'actrice, notre parti pris a été de le laisser de côté, pour nous concentrer sur les représentations qu'en donnent des arts sinon concurrents, du moins différents. Il en va de même pour les photographies de presse qui participent de ce qu'on peut considérer globalement comme les images du cinéma. Nous avons également choisi de ne pas aborder les œuvres poétiques, qui posent des questions génériques différentes, ainsi que les œuvres dites paralittéraires, des biographies aux «fan-fictions ». 


\section{Le genre sexuel au centre des genres littéraires et artistiques?}

En traitant du cinéma, romanciers et artistes semblent de près ou de loin, s'inscrire dans des genres esthétiques qui ne sont pas exempts de déterminations de genre : romans et œuvres plastiques traitent en effet de la comédienne et de l'actrice au détriment de son homologue masculin. L'actrice est ainsi un personnage d'élection du roman de la fin du XIX et du début du $\mathrm{Xx}^{\mathrm{e}}$ siècle, et conduit certains critiques à parler du « genre de l'actrice » ou des « romans de l'actrice $»^{5}$ à propos de Nana de Zola, $L a$ Faustin des frères Goncourt, Bruges-la-morte de Rodenbach, ou encore The Tragic Muse d'Henry James et Die Rahl d'Hermann Bahr. Dans un contexte global d'interrogation du roman sur la féminité ${ }^{6}$, l'actrice est une figure plus singulière que d'autres personnages féminins, car elle appartient au théâtre, un art qui fascine l'Europe à cette période, et a une image de femme trompeuse. Cependant, depuis lors, le théâtre a subi la concurrence du cinéma, devenu art reconnu et industrie florissante. Au même titre que les comédiennes, les actrices ont inspiré les romanciers dans le monde anglophone, puis en France, dans la lignée des romans de l'actrice et d'un autre genre, apparu aux États-Unis, le roman d'Hollywood'7 . Ainsi, en 1981, Doris Grumbach s'inspire de Marilyn Monroe et de Frances Farmer pour construire 1'hérö̈ne de The Missing Person ${ }^{8}$. C'est également Marilyn Monroe qui inspire Joyce Carol Oates en 2000, dans Blonde 9 . La même année, une actrice à la notoriété moindre devient elle aussi une héroïne de roman avec Ingrid Caven ${ }^{10}$, écrit par son compagnon, Jean-Jacques Schuhl.

5. Respectivement Sylvie Jouanny, dans sa thèse : L'Actrice et ses doubles, figures et représentations de la femme de spectacle à la fin du XIXe siècle, Genève, Droz, 2002, et Corinne François, dans une autre thèse, comparatiste celle-ci : Les " Romans de l'actrice » : 1880-1916 (Domaines germanique, anglo-saxon et français), Thèse de doctorat en littérature comparée, dir. Yves Chevrel, Paris, Université Paris Sorbonne, 2004. Sylvie Jouanny, qui analyse les romans comme les mémoires et les biographies, parle plutôt d'un « sous-genre à l'intérieur des genres littéraires [qui] témoigne de combinaisons formelles et sémantiques récurrentes, qui forment implicitement un modèle, qu'il soit suivi ou transgressé » (Sylvie Jouanny, op. cit., p. 16). Le « genre de l'actrice » est ainsi pour elle une notion transversale et transgénérique. 6. Cf. les travaux de Mireille Dottin-Orsini, Cette femme qu'ils disent fatale. Textes et images de la misogynie fin-de-siècle, Paris, Grasset et Fasquelle, 1993.

7. Cf. Bruce Chipman, Into America's Dream-Dump. A Postmodern Study of the Hollywood Novel, Lanham-New York-Oxford, University Press of America, 1999, p. xi : « The Hollywood Novel is defined as any novel set in Hollywood or centered on movie-making that deals with Hollywood as a revealing cultural phenomenon » (« Le roman d'Hollywood se définit comme un roman se déroulant à Hollywood ou centré sur le cinéma, qui traite d'Hollywood comme un phénomène culturel révélateur »).

8. Doris Grumbach, The Missing Person, New York, Norton, 1993 [1981]. Ce livre n'étant pas traduit en français, toutes les traductions des passages que nous donnerons seront de notre fait. 9. Joyce Carol Oates, Blonde, a Novel, New York, Ecco Press, 2001 [2000], traduit de l'américain par Claude Seban sous le même titre, Paris, Stock, Livre de Poche, 2000.

10. Jean-Jacques Schuhl, Ingrid Caven, Paris, Gallimard, coll. « Folio » (édition augmentée), 2002 [2000]. 
Bien que la carrière d'Ingrid Caven soit marquée à la fois par le cinéma et par la chanson, elle est bien « une actrice de cinéma sur scène ${ }^{11}$ ». Enfin, en 2006, c'est encore Marilyn Monroe qui inspire Michel Schneider dans Marilyn dernières séances ${ }^{12}$, histoire des relations de la star avec son psychanalyste. La production romanesque francophone et anglophone récente témoigne donc d'un intérêt notable pour les figures d'actrices de cinéma, qui deviennent protagonistes d'œuvres fictionnelles, plus spécifiquement des « romans biographiques » dans le cas de Blonde et d'Ingrid Caven.

Dans les arts plastiques, les représentations d'actrices ressortissent à différentes traditions. La plus ancienne pourrait être celle du portrait d'actrice en Angleterre à la fin du XVIII ${ }^{\mathrm{e}}$ siècle, époque où l'actrice acquiert une visibilité et un rôle social sans précédent et sans commune mesure avec celui de l'acteur ${ }^{13}$. Cette période semble ainsi fonder et légitimer le portrait d'actrice, exemplifié par Gainsborough ou Reynolds. Avec l'invention de la photographie, danseuses et comédiennes n'hésitent pas à poser pour des photographies de petit format, et ces images, vendues à bon marché, se collectionnent ${ }^{14}$. Avec le développement des techniques d'impression, la photographie a été incluse dans les journaux et les magazines, dont certains se sont spécialisés dans les actualités des vedettes de cinéma. Sans former un genre, ces images semblent donner une place particulière à l'actrice plutôt qu'à l'acteur, ce qui s'expliquerait selon Edgar Morin, par le lectorat de ces magazines, majoritairement féminin. Peut-être cette prééminence de l'actrice doit-elle s'interpréter également à la lumière d'une histoire de l'art plus vaste, faisant de l'actrice un avatar moderne de la muse. À l'époque contemporaine, les arts plastiques ${ }^{15}$ se sont emparés de la figure de l'actrice, notamment avec le Pop Art qui a récupéré les images véhiculées par les médias de masse. Les œuvres les plus emblématiques sont celles d'Andy Warhol, parmi lesquelles on peut évoquer Gold Marilyn ${ }^{16}$, qui isole la face de l'actrice sur un fond d'or, tout comme

11. Jean-Jacques Schuhl, op. cit., p. 198.

12. Michel Schneider, Marilyn dernières séances, Paris, Gallimard, coll. « Folio », 2006.

13. Robyn Aleson (ed.), Notorious Muse : the Actress in British Art and Culture, 1776-1812, New Haven, Yale University Press, 2003.

14. David Mayer, « The Actress as Photographic Icon : from Early Photography to Early Film », dans Maggie B. Gale et John Sotkes (eds.), The Cambridge Companion to the Actress, Cambridge, Cambridge University Press, 2007, p. 74-94. En France, Sarah Bernhardt est la plus représentée, photographiée notamment par les Nadar. Cf. Noëlle Guibert (dir.), Portrait(s) de Sarah Bernhardt, Paris, Bibliothèque Nationale de France, 2000.

15. Pour désigner les œuvres photographiques, les sérigraphies et les œuvres d'art vidéo de notre corpus, nous préférons le terme d' " arts plastiques », qui semble moins réducteur que celui d' « art visuels », car les réalités qu'ils désignent tous deux ne contiennent pas que du visuel, même si celui-ci prime souvent.

16. Andy Warhol, Gold Marilyn, 1962, acrylique, sérigraphie à l'encre, peinture dorée et peinture en spray sur toile, 211,5 x 144,8 cm, New York, Museum of Modern Art, Cat. 248. 
Round Marilyn ${ }^{17}$, qui prend en outre la forme d'un tondo. Marilyn's Lips ${ }^{18}$ présente l'originalité de fragmenter le visage et de donner à cette bouche quelque chose de monstrueux. Parmi les œuvres consacrées à Liz Taylor, Men in her life $e^{19}$ peut être lu comme un témoignage biographique, comme Silver Liz as Cleopatra ${ }^{20}$, qui dépeint l'actrice dans un de ses rôles les plus célèbres. Enfin, les Ten Lizes ${ }^{21}$ semble être l'une des œuvres les plus représentatives des portraits d'actrice de Warhol. Les œuvres de cet artiste font entrer de manière décisive dans l'art les images que le cinéma produit : celles des films, mais aussi des photographies de studio ou de presse. Une quinzaine d'années plus tard, Cindy Sherman réalise la série des Untitled Films Stills ${ }^{22}$. Elle imite les films stills ${ }^{23}$ de vieux films en devenant ellemême l'actrice et le personnage. La représentation de l'actrice se double ainsi de la représentation de soi dans ces 69 photographies en noir et blanc. En 1996, la photographe Zoe Leonard et la cinéaste Cheryl Dunye créent le personnage de Fae Richards, actrice américaine noire et lesbienne, qu'elles font naître en 1908 et mourir en 1973. The Fae Richards Archive ${ }^{24}$ est ainsi un ensemble de 73 photographies qui présentent le récit en images de la vie de l'actrice. La figure de l'actrice est également omniprésente dans l'œuvre de Douglas Gordon, notamment dans une série exclusivement consacrée à des interprètes féminines : Self Portrait of You + Me (James Bond Girls) ${ }^{25}$. L'artiste y utilise des photographies des James Bond Girls,

17. Andy Warhol, Round Marilyn, 1962, sérigraphie à l'encre et peinture dorée sur toile, 45,1 cm (diamètre), collection particulière, Cat. 283.

18. Andy Warhol, Marilyn's Lips, 1962, acrylique, sérigraphie à l'encre et crayon sur toile, $210,2 \times 209,2 \mathrm{~cm}$ (toile noire et blanche); 210,2 x 205,1 cm (toile en couleurs), Washington DC, Hirshhorn Museum and Sculpture Garden, Smithsonian Institution, Cat. 285.

19. Andy Warhol, Men in her life, 1962, sérigraphie à l'encre et crayon sur toile, 208,3 x 208,3 $\mathrm{cm}$, collection particulière, Cat. 301.

20. Andy Warhol, Silver Liz as Cleopatra, 1963, peinture argentée, sérigraphie et crayon sur toile, 208,3 x 165,1 cm, Toronto, Art Gallery of Ontario, Cat. 381.

21. Andy Warhol, Ten Lizes, 1963, sérigraphie à l'encre et peinture en spray sur toile, 200,2 x 564,5 cm, Paris, Musée national d'art moderne, Centre Georges Pompidou, Cat. 450. 22. Cindy Sherman, Untitled Film Stills, 1977-1980, 69 photographies argentiques en noir et blanc, 14 à 19,2 cm (hauteur) x 21,4 x 24,1 cm (largeur), New York, Museum of Modern Art. Pour faire référence aux Untitled Films Stills, nous indiquerons ici l'année et le numéro de la page du catalogue dans lequel ils figurent : David Frankel (éd.), Cindy Sherman. The Complete Untitled Film Stills, cat. expo., juin-septembre 1997, New York, Museum of Modern Art.

23. Les film stills sont des photographies utilisées le plus souvent pour la publicité d'un film, images issues de la pellicule, photogrammes, ou scène reconstituée par les acteurs après une prise.

24. Zoe Leonard et Cheryl Dunye, The Fae Richards Photo Archive, 1996, 78 photographies argentiques en noir et blanc et 4 tirages chromogéniques en couleurs, dimensions variables, New York, Whitney Museum of American Art.

25. Douglas Gordon, Self Portrait of You + Me (James Bond Girls), 2006, 44 photographies argentiques (17 en noir et blanc, 17 en couleurs), miroirs et fumée, $83,3 \times 73 \times 7 \mathrm{~cm}$, Londres, Gagosian Gallery. 
en brûle les yeux et la bouche et appose un miroir derrière la photographie. On peut enfin mentionner Sophie Calle qui, dans Prenez soin de vous, fait lire ou « interpréter » à cent sept « femmes » (cent cinq femmes et deux animaux femelles) la lettre de rupture qu'elle a reçue. Parmi ces femmes se trouvent des actrices, dont le nombre élevé - dix-sept - témoigne d'une sur-représentation, qui justifie qu'on les analyse séparément. Force est de constater qu'en art, les représentations d'actrices sont plus nombreuses que dans le roman et l'on pourrait évoquer également les œuvres de Michel Journiac, de Bettina Rheims, de Yasamusa Morimura, de Pierre Bismuth ou encore de Brice Dellsperger et Thomas Lélu.

\section{Des représentations genrées?}

Aborder la place du genre dans ces représentations requiert de s'interroger sur le genre même de celui qui représente et propose un certain regard sur la féminité, regard qui ne semble pas être le même chez les hommes et chez les femmes. Chez Schuhl et Schneider, la narration se fait à travers la médiation du regard masculin. Charles, qui partage la vie d'Ingrid Caven, est un double de l'auteur. Dans Marilyn dernières séances, le narrateur est un journaliste qui évoque la relation de l'actrice avec son psychanalyste. Fondé sur la parole, comme toute analyse, ce rapport peut dupliquer, en son sein même, le récit. On peut ainsi voir dans le psychanalyste Greenson, qui met en mots l'actrice, un autre narrateur, rappellant d'ailleurs que l'auteur lui-même est psychanalyste. Dans les romans écrits par des femmes, la narration est plus complexe : le narrateur est le plus souvent omniscient, donc a priori non genré. Il adopte parfois les points de vue de différents personnages. Cette polyphonie narrative pourrait indiquer le refus de rendre compte de manière univoque de l'actrice et de sa féminité.

D'un point de vue thématique, le regard masculin, qui rappelle ici le « male gaze » cinématographique théorisé par Laura Mulvey invite peutêtre à trouver, chez les romanciers, une stéréotypie plus grande de la figure de l'actrice : sa représentation tend ainsi vers « l'idéal féminin », et n'est, chez Schuhl, pas compensée, comme chez les romancières, par une présence du corps défétichisé. Chez elles, le donné naturel de la différence des sexes apparaît crûment dans l'évocation de la sexualité féminine et de l'enfantement, ou plutôt de l'échec de l'enfantement. On remarque également dans The Missing Person la maladie de la doublure de l'héroïne : un cancer du sein, qui donne lieu à une amitié féminine alors que ce sentiment semble souvent éclipsé par l'amour hétérosexuel dans les autres textes. On pourrait faire de cet aspect un trait d'une écriture genrée, qui rappelle d'ailleurs le féminisme de Grumbach et l'homosexualité féminine souvent abordée dans ses romans ${ }^{26}$. Un autre motif qui différencie romanciers et romancières est

26. Cf. The Ladies, New York, E. P. Dutton, 1984 et The Magician's Girl, New York, MacMillan, 1987. 
celui du couple formé par l'actrice et l'écrivain. On le rencontre magistralement chez Schuhl :

La vieille histoire qui fascine les foules : l'écrivain et l'actrice, ou la chanteuse, D'Annunzio et la Duse, Miller et Monroe, Gary et Seberg, Shepard et Jessica Lange, Philip " Portnoy » Roth et Claire « Limelight» Bloom, les noces du verbe et de la chair $[\ldots]^{27}$.

Si les exemples sont nombreux, le traitement semble ici relever complaisamment d'un cliché qui réduit la femme actrice à la muse et à la « chair », en fait le corps d'un couple dont la tête pensante est l'écrivain.

Dans le cas des artistes, les oppositions de genre sont plus subtiles; il semble que les artistes femmes fassent du genre un objet d'interrogation à part entière et non un simple aspect de la représentation. Ainsi peut-on lire les photographies de Sherman où l'artiste est le modèle et mène une réflexion non seulement sur les images féminines stéréotypées - comme celle que mène Gordon - mais aussi sur le sujet féminin en tant que consommateur de ces images et les rapports possibles d'identification. Dans l'œuvre de Sophie Calle, l'entre-femmes fait partie du projet. Geste inverse de celui que subissent les femmes dans la société, l'exclusion du sexe masculin, la confiscation de sa parole et l'affirmation d'un savoir des femmes, autorisent une lecture féministe. Enfin, l'idée de communauté féminine apparaît également dans la série de photographies de The Fae Richards Archive, puisque plusieurs photographies montrent l'actrice avec ses compagnes. La seconde, très masculine, illustrant l'identité butch $^{28}$, propose une vision de la féminité différente de celle qui est incarnée par l'actrice et questionne les frontières du genre.

\section{Le genre représenté : le brouillage des signes}

Les œuvres de Joyce Carol Oates, de Douglas Gordon, de Michel Schneider ou de Sophie Calle n'ont apparemment pas vocation à faire école ou à créer un style particulier, mais en dépit de cette apparente diversité, ces œuvres ont toutes la particularité plus ou moins revendiquée comme telle, de représenter une ou plusieurs actrices et de faire du genre un élément central de cette représentation, en brouillant les signes ou en les inversant.

L'actrice se définit avant tout par son métier : jouer un personnage devant un dispositif d'enregistrement de l'image puis, avec les progrès techniques du cinéma, du son. Être actrice, c'est donc réaliser une performance de jeu dont le film garde la trace. Les œuvres romanesques et plastiques dépeignent ce qui se passe sur l'écran, mais aussi l'avant et

27. Jean-Jacques Schuhl, op. cit., p. 87.

28. La critique queer a souvent repris les termes utilisés par les lesbiennes elles-mêmes pour se différencier : on distingue ainsi la lesbienne butch, qui adopte les codes de la virilité, de la lesbienne $f e m$, qui répond aux codes de la féminité. 
l'après, comme le jeu devant la caméra ou la vie quotidienne dans le monde du cinéma. Les vidéos de Sophie Calle créent un espace d'improvisation qui semble donner à voir l'essence même du métier d'actrice, le jeu se faisant, avec ses dangers et ses ratés. La définition de l'actrice par son jeu, aussi minimale soit-elle, n'est cependant pas dépourvue des déterminations du genre, qui apparaissent à travers les romans dans un questionnement sur la capacité de jouer. Ainsi les personnages qui côtoient Marilyn Monroe dans Blonde ne savent pas si elle est « une vulgaire grue valant à peine mieux qu'une pute ou qu'une actrice de porno » (« a common tramp hardly better than a hooker or pornfilm performer 》) ou " une actrice née, à ce titre une sorte de génie » (« a born actress, a natural, in that way a kind of genius ») $)^{29}$. Grumbach et Oates montrent une Marilyn Monroe et une Franny Fuller qui s'efforcent de se métamorphoser en leurs personnages, voulant pousser à l'extrême l'identification, parfois jusqu'à la métamorphose au plus intime de l'être, quand le sexe de Norma Jean gonfle lorsqu'elle interprète le rôle de Rose Loomis, personnage de femme en révolte contre l'ordre masculin ${ }^{30}$. Cependant, dans Marilyn dernières séances, le personnage de Mankiewicz s'oppose à cette identification marquée par les méthodes de Broadway : «Elle [Monroe] savait ça d'instinct et toutes ses années de conversion religieuse à "la Méthode" Strasberg n'ont pas réussi à la gâcher ${ }^{31}$. » Le mot « instinct» rapproche cependant cette analyse du point de vue opposé car le questionnement sur le jeu n'est jamais envisagé en termes professionnels : le métier est naturalisé, l'actrice « déprofessionnalisée ». Il s'agirait d'une différence sociologique entre l'acteur et l'actrice ${ }^{32}$ - plus volontiers considérée comme une célébrité que comme une professionnelle - qui justifierait dans les romans des expressions telles que " génie » « naturel» ou 《actrice née $»^{33}$. La question de la maîtrise ou de l'absence de jeu n'est jamais tranchée, et semble constitutive de l'ambiguïté même de l'être de l'actrice dans les romans.

En effet, l'identification de l'actrice avec son personnage semble parfois telle qu'elle brouille son identité, au profit d'une entité tierce et ambiguë, qu'on pourrait appeler sa persona. À ce titre, le pseudonyme et les oscillations de la désignation se font l'emblème de cette identité instable. Dans The Missing Person, Fanny Marker prend le pseudonyme de Frances Fuller et devient, une fois célèbre, Franny Fuller. Dans les arts plastiques, c'est cette persona qui est convoquée, comme dans Silver

29. Joyce Carol Oates, op. cit., p. 328, trad. p. 500-501.

30. Ibid., p. 505. Ce soin extrême apporté à l'identification n'est pas sans suggérer une certaine folie, que le personnage de Greenson évoque d'ailleurs à propos d'une autre actrice, Vivien Leigh, dans Marilyn dernières séances, op. cit., p. 143.

31. Michel Schneider, op. cit., p. 376.

32. Cf. Karen Hollinger, The Actress : Hollywood Acting and the Female Star, London, Routledge, 2006.

33. Joyce Carol Oates, op. cit., p. 328, trad. p. 500-501. 
Liz as Cleopatra. L'image provient d'un des rôles les plus célèbres de l'actrice, pour sa performance, comme pour l'histoire même du tournage : c'est à cette occasion qu'elle se lie avec Richard Burton, alors qu'elle est mariée à Eddie Fisher. Dans cette double représentation d'une femme célèbre, belle et "fatale », le rôle de la reine d'Égypte, séductrice, coïncide avec l'identité de l'actrice à la ville, et les deux s'incarnent dans la persona de l'actrice.

Dans la construction de cette identité, visage et corps tiennent une place centrale. Pris dans une cosmétologie "hypergenrée ", ils posent le problème de l'image des femmes dans l'industrie culturelle et dans la société. Le cinéma les traite comme un ensemble de signes, qui sont eux-mêmes pris dans des significations qui les dépassent, comme le montre le Barthes de Mythologies au sujet du visage de Garbo ${ }^{34}$. Le visage est surface plastique, matière expressive et signe d'identité. Les romanciers et les artistes montrent que cette identité est complexe et multiple. Elle est à la fois naturelle, et fabriquée par le maquilleur, qui la fait renaître à chaque séance, et accentue les traits du genre. Dans Blonde $e^{35}$ comme dans Ingrid Caven, le maquilleur magicien métamorphose l'actrice, qui disparaît « derrière le voile cosmétique, sous le film $^{36}$ ». Dans les Untitled Film Stills de Cindy Sherman, l'usage du maquillage est spectaculaire. Il s'éloigne du maquillage traditionnel de cinéma, puisque la métamorphose est telle qu'elle semble oblitérer le visage de l'artiste pour créer celui de l'actrice. En revanche, plus que dans les autres œuvres, le travail de Sherman questionne les potentialités expressives du visage, en mettant en scène une sémiologie de l'expression en images.

Pris entre les signes des costumes et la nudité, le corps est un enjeu de signification particulier. C'est également Sherman qui met le mieux en évidence les potentialités du costume et son fonctionnement, en résonance avec d'autres éléments : pose, décor, maquillage construisent un personnage, comme la figure du numéro $35^{37}$ : dans un coin de pièce, contre une porte sale se tient une femme, vue en contre-plongée, adoptant une pose et un regard de défi. Elle porte une robe-blouse à motifs vieillots, et un tablier aux motifs différents. Sur sa tête, un foulard d'où sortent les mèches blondes de la perruque. Ses jambes sont couvertes de mi-bas chair, dont le pli apparait sous le genou. Les chaussures noires à talons sont lourdes. L'artiste-actrice incarne ici un personnage que composent ses vêtements : une femme de condition modeste, à la vie sans doute difficile. Femme travailleuse, occupée à des tâches ménagères, de la vie de laquelle l'âpreté convient au caractère décidé suggéré par la pose. Ce personnage fait penser aux films néoréalistes

34. Roland Barthes, « Le visage de Garbo », dans Mythologies, Paris, Seuil, coll. « Points », 1970 [1957], p. 70-72. Barthes fait du mythe une forme de l'idéologie.

35. Cf. particulièrement la belle scène de Blonde, p. 625-626, trad. p. 945.

36. Jean-Jacques Schuhl, op. cit., p. 16.

37. Cindy Sherman, op. cit., numéro 35, 1979, p. 135. Cf. illustration page suivante. 


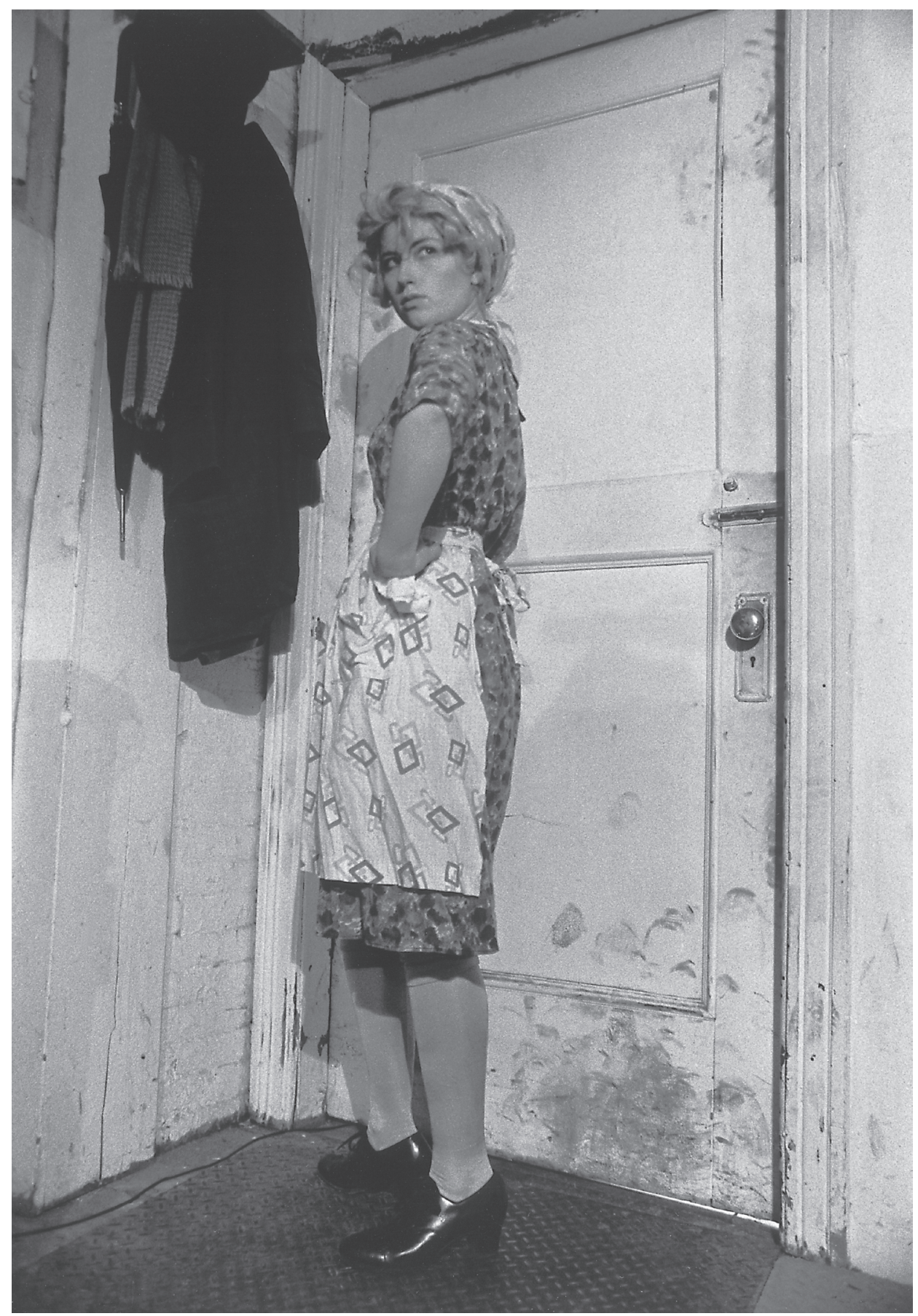

Cindy Sherman, Untitled Film Still, $n^{\circ} 35,1979$.

Black and white - photograph $-10 \times 8$ inches. Courtesy of the Artist and Metro Pictures. 
italiens, à tel point qu'on pourrait croire que c'est Silvana Mangano, la « mondine » de Riso Amaro (Riz Amer de De Santis, 1969) qui apparaît sur cette photo ou bien, d'après Cindy Sherman elle-même, Sofia Loren, dans un de ses rôles comme La Ciociara ${ }^{38}$ (La Paysanne aux pieds nus ou Two Women de De Sica, 1960). On remarque enfin d'autres vêtements, masculins, accrochés au porte-manteau, peut-être ceux d'un homme à qui semblerait destinée cette attitude défiante. On voit ainsi comment le personnage est identifié, campé, par ce que son corps revêt, en accord avec la manière dont le corps est posé au sein d'un décor.

Sous les costumes, le corps apparaît. Dans la lignée de la tradition artistique occidentale, le cinéma fait du corps féminin un objet de représentation particulier, idéalisé, manifestation d'une féminité exemplaire. Aux yeux de la société, le corps de l'actrice incarne ainsi un canon, comme celui de la «Blonde Bombshell» («Bombe blonde ») Marilyn Monroe, dont le corps est objet érotique. Ce corps est désigné par Grumbach comme objet du désir masculin : "The shadow of herself up there belonged to their fast, wet dreams ${ }^{39}$. " ("Son ombre là-haut sur l'écran appartenait à leurs rêves hâtifs et humides. ») Ce qu'on appelle le « glamour ${ }^{40}$ » est peut-être la qualité de ce corps parfait et désirable toujours chargé de signifier une sexualité idéale. C'est dans une logique de pastiche que Sherman rejoue ces mises en scène du «male gaze », comme dans le numéro $36^{41}$ : dans cette scène de déshabillage en contre-jour, la figure est rendue absente, parce que son visage est masqué, comme si l'actrice était réduite à la présence de son corps toujours objet.

Magnifié, ce corps fonde la transcendance de l'actrice, nouvelle déesse païenne de la société de consommation. Le terme de star reflète bien le statut supérieur et idéal de l'actrice dans les représentations sociales. Les romans s'en font l'écho, rappelant parfois la réussite du mythe du rêve américain à travers les «American Dream girls » que sont Franny Fuller et Marilyn Monroe. Dès lors, l'actrice a ses icônes, ce que suggère Warhol par l'emploi de la peinture argentée ou dorée et par une représentation frontale, comme dans l'art byzantin, qui semble concentrer la vénération sur la divinité réduite à l'archétype ${ }^{42}$. C'est cet archétype que la peinture

38. Cindy Sherman, op. cit., p. 8.

39. Doris Grumbach, op. cit., p. 58.

40. En anglais, le terme «glamour» désigne une capacité de séduction. Il est issu d'une déformation du terme grammar (grammaire), qui désignait l'apprentissage scolastique et par extension, des connaissances occultes. La variation du mot en écossais a donné gramarye, qui désigne la magie, l'enchantement. Le mot est en français un anglicisme, qui fait référence en particulier au charme des vedettes féminines d'Hollywood.

41. Cindy Sherman, op. cit., numéro 36, 1979, p. 90.

42. Remarquons que ce traitement n'a rien à voir avec celui réservé à Elvis Presley, représenté en action; cela pourrait rappeler l'un des ressorts de la fétichisation selon Laura Mulvey : la représentation de la femme sur un espace sans profondeur. 
répète en série, mais à l'aide de la technique : l'empreinte lumineuse de la photographie semble rejouer ces traces du divin que sont les icônes dites achéiropoiètes, «non faites de main d'homme », comme la Véronique ${ }^{43}$. Paradoxalement, les motifs de la blancheur et de la lumière font tendre la figure vers une pureté virginale : dans Marilyn dernières séances, le blanc est sans cesse évoqué, à travers les cheveux décolorés de l'actrice, comme dans son cocktail favori, l' «Ange blanc ». C'est ainsi l'actrice elle-même qui semble devenir un ange, rappelant le nom même de la ville de Los Angeles : «Here was an angel, an angel with breasts and hips 》 («c'était un ange, mais un ange avec hanches et poitrine ») écrit Oates ${ }^{44}$. Tous les romanciers insistent sur le rayonnement de l'actrice à l'écran. Dans Ingrid Caven, le blanc relie l'histoire intime au métier d'actrice dans une scène où l'héroïne subit une attaque de la maladie de peau qui la ronge ${ }^{45}$. La blancheur des draps de la chambre rappelle les tissus blancs de l'église, le Leintuch et l'écran du cinéma, le Leinwand, réunis dans une même sacralité, dont l'actrice semble ici être dépendante. Le black-out de la crise rejoue le « noir et blanc » du cinéma, comme si la puissance du blanc était augmentée par la présence du noir. Dans The Fae Richards Photo Archive néanmoins, le blanc semble inversé en son contraire, puisque l'idole du cinéma est noire, dans un geste critique envers l'idéalisation candide de l'actrice.

Cette sacralité est cependant mise à mal : la déesse de l'amour est condamnée à l'échec, comme l'illustre Warhol qui déplie le fil narratif de la vie amoureuse de Liz Taylor : le titre Men in her Life et non «Men of her life », pourrait porter un éclairage irrévérencieux sur la vie amoureuse de l'actrice. La star reste ainsi « hérö̈ne d'amour », mais parce qu'elle incarne les échecs plus que la réussite du sentiment amoureux. Dans la tradition des romans de l'actrice, les œuvres littéraires se construisent en partie au fil des relations de l'héroïne. La représentation de l'actrice est donc double : elle souscrit au cliché de la « femme fatale » mais la montre ellemême malheureuse ${ }^{46}$. Dans Marilyn dernières séances, le charme semble même rompu, puisque l'idole réduite à un corps offert ne suscite que le " dégoût ${ }^{47}$ » de Greenson. Cette déchéance va de pair avec la frigidité de l'actrice dans The Missing Person ${ }^{48}$. Parce qu'elle éveille le désir, l'actrice est, dans les représentations sociales, plus proche de la « putain » que de la « maman », pour reprendre les termes utilisés par Jean Eustache. L'actrice du second $\mathrm{Xx}^{\mathrm{e}}$ siècle n'est donc pas si éloignée d'une Nana, et l'image de

43. Le nom de cette icône vient de celui de sainte Véronique qui tendit au Christ un linge sur lequel, par simple contact avec son visage, son image s'imprima miraculeusement.

44. Joyce Carol Oates, op. cit., p. 377, trad. p. 573.

45. Jean-Jacques Schuhl, op. cit., p. 80.

46. Plaisir iconoclaste que retrouverait le lecteur des tabloïds?

47. Michel Schneider, op. cit., p. 230.

48. Doris Grumbach, op. cit., p. 81. 
la prostituée lui colle à la peau. Dans les romans tout particulièrement, le corps de la maternité et son échec entrent en contraste avec le corps idéal de l'actrice. Dans The Missing Person, une fausse couche vient rompre les aspirations de l'actrice à être mère, comme dans Blonde : " her living baby hemorrhaged out of her in a mass of clots \& clumps like something but partially digested 》 (« un flot de sang expulsait son bébé vivant de son corps par bouts et par morceaux comme quelque chose de mal digéré »4) La traduction rend ici peu compte de la crudité des expressions, parmi lesquelles les termes «hemorrhaged » et « digested » renvoient sans fard à la vie du corps. On remarque en outre l'expression «living baby », qui présente la fausse couche dans toute sa violence, d'autant que le groupe est en anglais sujet, et non objet, du verbe « hemorrhaged », ce qui associe mère et enfant dans un même saignement et dans une même douleur.

Déchu, ce corps magnifié et exhibé est un capital économique si perfectionné qu'il semble devenir étranger. « Robot créé par le studio ${ }^{50}$ » l'actrice ne possède plus son corps, rappelant peut-être ainsi le concept freudien d' « inquiétante étrangeté ». Dans un portrait de Franny Fuller par sa doublure, l'impression d'étrangeté est concentrée dans les deux parties du corps qui en condensent la féminité, les seins et les hanches, que leur pouvoir symbolique particulier semble avoir rendus autonomes. L'actrice devient alors : "Comme une sorte de grand jouet, [...] une femme magnifique mais à l'aspect curieusement mécanique. » ("Like some great toy, $[\ldots]$ a magnificent but curiously mechanical woman $\left.{ }^{51} . »\right)$

\section{États-limites : inversion et destruction}

Dans leur entreprise de questionnement des signes de la féminité de l'actrice, artistes et romanciers vont même jusqu'à inverser ces signes, à contre-courant des représentations sociales : l'actrice sex-symbol devient alors une figure masculine. Dans The Fae Richards Photo Archive, l'actrice apparaît à plusieurs reprises travestie, rappelant son homosexualité, d'une manière quelque peu conventionnelle qui rappelle par exemple Marlène Dietrich de Cours brûlés (Morocco, 1930) de Sternberg. Les romans semblent cependant aller plus loin. C'est ainsi que Franny Fuller est d'abord prise pour un homme par les amis de son futur mari : " "That guy back there is drunk already. Pretty early in the day)." " ("Ce type là-bas est déjà saoul. Drôlement tôt dans la journée" ») s'exclament-ils, avant de se

49. Joyce Carol Oates, op. cit., p. 624, trad. p. 943.

50. Ibid., p. 633, trad. p. 957 : «MARYLIN MONROE was a robot designed by The Studio. » («Marylin Monroe était un robot créé par le studio. »). NB : les capitales ne sont pas une erreur, mais la typographie de l'édition originale, non respectée dans la traduction, que nous avons cependant choisi de suivre.

51. Doris Grumbach, op. cit., p. 149.

52. Ibid., p. 122. 
rendre compte que cette personne affaissée sur le bar est une jeune femme. Le personnage du travesti apparaît dans trois des quatre romans analysés, comme une présence inquiétante pour l'actrice. Dans Blonde, le numéro du travesti devient cauchemar, car l'actrice souffre de se retrouver dans ce reflet grotesque :

The dancer struts amid swirling smoke \& deafening music holding her enormous breasts like foam rubber, neon-pink nipples the size of grapes \& next the pleated skirt is torn off \& tossed \& she's shimmying her plump ass \& turns her back to the screaming audience stoops \& spreads cheeks — Ohhhhh! the audience groans, yells - the dancer now nude gleaming in powdery - caked oily - white sweat on her pimply back \& turning at last in triumph to reveal the long slender penis taped to the shaved pubis with flesh-colored adhesive $[\ldots]$ \& now the crowd in Club Zuma has truly gone crazy, screaming at the dancer \& frantic bobbing penis semi-erect

MARI-LYN! MARI-LYN! MARI-LYN!

La danseuse se pavane dans les spirales de fumée et la musique assourdissante en tenant ses énormes seins rebondis caoutchouc mousse, mamelons rose fluo gros comme des grains de raisin \& ensuite c'est la jupe plissée qui est arrachée $\&$ jetée $\&$ elle tortille son cul dodu \& tourne le dos aux spectateurs en délire se baisse \& écarte les fesses... Ohhhh! le public grogne, s'égosille... La danseuse nue maintenant une sueur mêlée de poudre luisant blanche $\&$ huileuse sur son dos boutonneux \& elle se retourne enfin triomphante pour révéler le long pénis fin collé au pubis rasé par un adhésif couleur chair [...] \& la foule du Club Zuma maintenant complètement déchaînée hurle au danseur \& à son pénis frénétique à demi dressé

MARI-LYN! MARI-LYN! MARI-LYN! $!^{53}$

La dernière phrase, qui accumule les propositions, mime tout particulièrement le suspens de la scène et sa tension. La performance du travesti, désigné par le mot «dancer », sans marque de genre en anglais, joue sur la reprise outrancière des signes du corps de l'actrice, qui provoque le désir et le rire. La scène est insupportable pour Marilyn Monroe, qui se sent ridiculisée et se rend peut-être compte, face à ce reflet grotesque, qu'elle est une construction destinée au désir masculin, révélé in fine par l'apparition du pénis ${ }^{54}$. Si l'on considère, avec Judith Butler, que le travesti est celui qui accomplit en permanence le genre, peut-être Marilyn Monroe, par sa féminité exacerbée, est-elle bien ce « travesti suprême ${ }^{55}$ », celui qui, plus que tout autre, performe le genre à chaque instant de son

53. Joyce Carol Oates, op. cit., p. 671, trad. p. 1014.

54. Une photographie de Yasumasa Morimura datant de 1996 ressemble curieusement à cette scène : on y voit une Marilyn vêtue de noir, dont la jupe s'envole et révèle un pénis en érection. Yasumasa Morimura, Self Portrait as an Actress, Black Marilyn, feuille ilfochrome acrylique, 120,2 x 95,25 cm, New York, Galerie Lurhing Augustine.

55. Joyce Carol Oates, op. cit., p. 614, trad. p. 926. 
apparition ${ }^{56}$. Dans Ingrid Caven, l'actrice n'est pas seulement imitée par un travesti décrit comme un dangereux vampire, mais en imite un ellemême, en lui empruntant sa voix ${ }^{57}$. Derrière l'idée de féminité idéale se cache peut-être ainsi une forme de masculinité que les romans semblent nous inviter à considérer.

Romans et œuvres plastiques semblent donc soumettre les signes de la beauté et de la supériorité du corps, et les signes du genre, à un brouillage qui va à l'encontre du corps glorieux de l'actrice à l'écran et des représentations sociales. Plus loin que le questionnement des signes, c'est une destruction presque iconoclaste que semble subir le visage. La série de Douglas Gordon est de ce point de vue la plus spectaculaire : à ces images commerciales qui offrent une beauté stéréotypée, l'artiste inflige une brûlure monstrueuse, faisant apparaître un crâne à la place du beau visage de Shirley Eaton ${ }^{58}$. De manière plus délicate, Zoe Leonard et Cheryl Dunye soumettent également leur actrice à une forme de défiguration : celle de la vieillesse, tandis que Sophie Calle filme certaines actrices sans fard, dans une lumière crue. Dans Blonde, la bouche maquillée devient «con»: les lèvres, véhicules de la parole et, partant, de la pensée, ne sont plus qu'une métonymie du sexe : "and her face, that's a special kind of cunt, the wet red mouth, the tongue " ("Et son visage, qui est un autre genre de con. La bouche rouge humide, la langue $»)^{59}$. Le « devenir-con» de la bouche ou la « régression bucale ${ }^{60}$ » infligée à l'actrice semble être ici le revers de l'érotisation excessive de son corps. Dans Marilyn's Lips ${ }^{61}$, Warhol fait de la célèbre moue de désir, répétée et isolée, un motif inquiétant, que les variations de la sérigraphie semblent animer. Plutôt que Vénus, Marilyn Monroe apparaît alors sous les traits de Méduse. Ainsi Schneider citet-il Mankiewicz dans Marilyn dernières séances : « [N]ous n'avons pas compris que la face de Méduse qu'elle nous laissait voir était un écran sur lequel nos désirs se projetaient, mais qu'ils ne traversaient pas ${ }^{62}$. » L'actrice fascinante saisit de stupeur, mais reste elle-même indifférente ${ }^{63}$.

56. Nous utilisons ici le terme «performer » en écho aux théories de Judith Butler qui théorise la performativité du genre (gender) chez le travesti, lequel accomplit et questionne de manière subversive le genre dans ses apparitions. Cf. Judith Butler, Gender Trouble, New York, Routledge, 1990.

57. «Chez qui tu trouves finalement ce déclic? Chez une espèce de trav' furieusement décadent [...] », Jean-Jacques Schuhl, op. cit., p. 359.

58. Douglas Gordon, Self Portrait of You + Me (Shirley Eaton), 2006, photographie argentique en couleurs, fumée et miroir, 83,3 x $73 \times 7 \mathrm{~cm}$, Londres, Gagosian Gallery.

59. Joyce Carol Oates, op. cit., p. 347, trad. p. 530.

60. L'expression de Georges Bataille est reprise par Thierry Grillet dans le catalogue de l'exposition Portraits/Visages. Sylvie Aubenas et Anne Biroleau (éd.), Portraits/Visages, cat. expo., octobre 2003 - janvier 2004, Paris, Bibliothèque Nationale, p. 14.

61. Andy Warhol, Marilyn's Lips, ceuvre citée.

62. Michel Schneider, op. cit., p. 377.

63. Rappelons que le latin traduisait le grec phallos par fascinus, dont dérive le terme fascination. 
La tête coupée des actrices de Warhol, leur maquillage devenu masque monstrueux, semblent en faire deux nouvelles Gorgones et justifier ainsi le commentaire de Kristeva : « [...] qui ne connaît la tête de Marilyn Monroe mise en série, ou sa bouche prélevée et multipliée par Andy Warhol : la plus séduisante des stars à l'usage de milliards de téléspectateurs, à moins que ce ne soit le suicide au bord des lèvres ${ }^{64}$ ? » On remarque d'ailleurs que l'effet est accentué en 1964, lorsque Warhol reproduit une série de Marilyn dont il rogne le cou, comme pour mieux rendre autonome cette tête décapitée ${ }^{65}$.

Entre images et mots, la figure de l'actrice est réinvestie par des artistes et des romanciers qui font du genre un élément central de leurs représentations. Par leur geste, ils s'inscrivent dans une histoire générique elle-même « genrée » car ces portraits et ces romans semblent privilégier la figure féminine. Le genre est également un critère qui peut différencier ceux-là mêmes qui représentent, mettant en évidence une vision parfois plus stéréotypée chez les hommes. L'actrice, être réel et réalisation commerciale du cinéma, semble être l'incarnation d'une sexualité idéale, que son corps et les récits dont elle est riche ne cessent d'affirmer. C'est ce que semblent viser les représentations secondes en instaurant, au milieu de cetteaffirmation du genre sans équivoque, l'indécision des signes troublés : la déesse d'amour devient mère malheureuse, femme monstrueuse, ou « travesti suprême ». On peut ainsi lire dans ces œuvres une dimension critique par laquelle sont déconstruites ces figures parfois trop univoques. Au cœur de cette identité indécise, le genre de l'actrice se révèle alors comme une construction instable.

\author{
Thibaut Casagrande \\ École normale supérieure de Lyon
}

64. Julia Kristeva, Visions capitales, cat. expo., avril-juillet 1998, Paris, Musée du Louvre, avril-juillet 1998, p. 111.

65. Cf. Andy Warhol, Shot Blue Marilyn, 1964, sérigraphie à l'encre, peinture acrylique sur toile, 101,6 x 101,6 cm, Greenwich Connecticut, Brant Foundation, Cat. 1292. 